\title{
EFFECT OF LOW INTENSITY BODY WEIGHT TRAINING ON BALANCE IN OLDER ADULTS
}

\section{Sumayya Faiz shaikh ${ }^{1}$, Simran Parti ${ }^{* 2}$, Khyati Shah ${ }^{3}$, Ronika Agrawal ${ }^{4}$.}

${ }^{1}$ Associate professor, M. A. Rangoonwala College of Physiotherapy \& Research (MARCOPAR), Hidayatullah Road, Azam Campus,Camp, Pune, Maharashtra, India.

${ }^{* 2}$ Graduate, P.T, M. A. Rangoonwala College of Physiotherapy \& Research (MARCOPAR), Hidayatullah Road, Azam Campus,Camp, Pune, Maharashtra, India.

${ }^{3}$ Graduate, P.T, M. A. Rangoonwala College of Physiotherapy \& Research (MARCOPAR), Hidayatullah Road, Azam Campus, Camp, Pune, Maharashtra, India.

${ }^{4}$ Principal, M. A. Rangoonwala College of Physiotherapy \& Research (MARCOPAR), Hidayatullah Road, Azam Campus,Camp, Pune, Maharashtra, India.

\section{ABSTRACT}

Background: Balance is the component which is majorly altered in older adults with approximately $21.5 \%$ of the population over 65 years of age having some balance dysfunction, particularly in functional tasks like ambulation and climbing stairs. Impaired balance and functional mobility are major risk factors for falls.

Aims and objective: The aim of the study was to study the effect of low intensity body weight training on balance in older adults.

Procedure: 60 older adults between the age group 65 to 80 with the BBS score between 46-51 were included in this study. The outcome measures used to assess the balance were the Bergs balance scale (BBS) and Performance oriented mobility assessment scale (POMA). The intervention program consisting of low intensity bodyweight training was carried out for 4 days a week for 6 weeks.

Results: The results after period of 6 weeks showed statistically significant post scores of BBS and POMA $(p<0.05)$ respectively.

Conclusion: Hence the author concluded that low intensity body weight training for a period of as less as 6 weeks can result in improvement of balance in older adults.

KEY WORDS: Balance, bodyweight training, low intensity, older adults.

Address for correspondence: Dr. Simran Parti, Graduate, P.T, M. A. Rangoonwala College of Physiotherapy \& Research (MARCOPAR), Hidayatullah Road, Azam Campus,Camp, Pune, Maharashtra 411001, India. E-Mail: partsimran@gmail.com

Access this Article online

Quick Response code

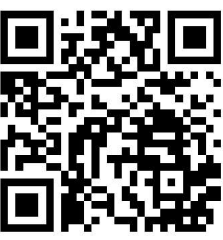

DOI: $10.16965 /$ ijpr.2019.116

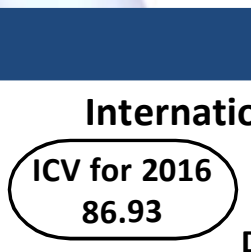

Journal Information

\section{BACKGROUND}

Aging or senescence is an inevitable progressive deterioration of physiological function with increasing age, demographically characterized by an age-dependent increase in mortality and decline of fecundity [1]. As far as the muscu- -loskeletal system is concerned, this unfavourable change usually takes the form of loss of the ability to develop force and an increase in stiffness of joints, ligaments and muscles [2]. Also the major consequences with aging are shrinkage of articular cartilage, 
vertebral discs, decreased ability to absorb shock, reduced range of motion, stiffer tissues, greater passive tension within tissues with more effort being required to move with loss of end range of motion [3]. The decline of muscle strength is to some extent a reflection of a reduction in muscle mass, and, a reduction in the number of motor units [2]. Little reduction in strength appears until age 40 or 50 , with minimal loss (10-20\% total) thereafter until age 6070. Persons in their 70's and 80's experience a more severe loss (30-40\%), with that loss being more pronounced in the muscles of the legs than in their hands and arms [4]. According to Baloh et al. young subjects have a higher dynamic balance capacity than ageing subjects. Getting older thus damages dynamic balance abilities. A fall, in ageing subjects is very often due to deterioration of balance and its consequences [5]. Decline in sensory (vision, vestibular and proprioception) and motor (strength, coordination, endurance) function and integration (response time, multi-task ability) have been identified as the major intrinsic factors contributing to falls. Previous research has shown that exercise such as strength, flexibility, balance training, and combinations of these activities delivered in a class situation or individually have increased strength, improved balance and improved functional ability [6].

Diminished balance ability is multifactorial. It can be due to degeneration of visual and vestibular sensory systems, degeneration of proprioception or impairment of central processing or a combination of these factors. Yet, studies have shown that lower extremity strength is a common factor associated with balance impairment in elderly fallers [7] Strength requirements have been reported for stepping and tripping to recover perturbed balance. The speed and organization of muscle force also contributes to balance problems in older age. Studies have proposed that older adults are at increased risk of injury, compared to young adults, due to differences in muscle strengths and speeds of muscle contraction. A reduced capacity for rapid force generation would limit the ability to respond quickly to a loss of balance and increase the risk of falling. Researchers also concluded that older adults, particularly those with a history of falls, are less successful in their balance recovery following perturbations, compared to young subjects, due to a slower generation of joint moments. Hence suggesting that some of the age-related changes to the balance system may be abated by maintaining a physically active lifestyle [8].

Age-related decline in strength is associated with loss of mobility, functioning and independence. It is clear that resistance training benefits older people by increasing strength and improving some aspects of functioning. Resistance training was defined as an exercise that involves the participant exerting effort against their body weight or external resistance [9].Studies have shown the use of high and moderate intensity strength training in improving the muscle strength in older adults $[10,11]$. High/ moderate intensity resistance training programs are associated with small, but real, risks of muscle or joint injuries and cardiovascular event; therefore, muscle training methods for elderly subjects need to be safe to perform [12]. As a result, the proportion of elderly people who do strength (resistance) training is currently low (about $10 \%$ to $15 \%$ ) [13]

Low intensity bodyweight training refers to using a relatively light load. It is a collective term, which refers to a practice of muscle training in which the load is increased and decreased extremely slowly [12]. Whereas, bodyweight training is any exercise that involves using the body as a means of resistance to perform work against gravity. Muscles work with the levers created through the bones and joints creating movement[14]. This training method has a positive effect on muscle hypertrophy and demonstrates an enhancing effect on muscle strength, even with load strength equivalent to $50 \%$ or less of the maximum muscle strength. However, studies on this method of training conducted on elderly subjects are in their accumulation stage and have predominantly been conducted on healthy elderly subjects, with the use of muscle training machines. Accordingly, a low intensity bodyweight training program that can be easily performed by elderly subjects at home is needed [12].

Therefore, the purpose of the study was aimed at finding the effect of low intensity body weight 
training on balance in older adults.

\section{MATERIALS AND METHODS}

The study design was pre-post interventional study. The sampling method used was convenient sampling. Sample size consisted of 60 older adults. The study was carried out in societies and old-age homes in Pune. The low intensity bodyweight training intervention was carried out for a total duration of 6 weeks. Inclusion criteria consisted of older adults aged 65 to 80 years of both the genders. The subjects with Bergs balance scale ranging from score 45-51 were included in the study. The older adults with any orthopaedic condition like recent fractures, acute case of osteoarthritis, lower limb surgeries, total knee replacement, etc. or any neurological involvement like stroke, Parkinsonism, diabetic neuropathy etc. or any cardiovascular condition like - myocardial infarction, angina, uncontrolled blood pressure,etc. were not taken into consideration. Performance oriented mobility assessment (POMA) and Bergs balance scale (BBS) were used as the outcome measures.

Procedure: After the approval of the Institutional Ethical Committee, on the basis of inclusion and exclusion criteria, 60 older adults were included in the study. The procedure was then explained to the subjects and their doubts regarding the same were cleared and a written consent was taken from all the subjects before starting the intervention. The intervention was conducted for 4 days a week for a duration of 6 weeks with progression of exercises after the first 3 weeks. Warm up and cool down exercises were given for 5 minutes before and after the session respectively. Blood pressure (BP) readings were recorded before and after the session. Sufficient rest period between the exercises was given and the subjects were instructed to inform if they likely experienced any discomfort during the session. Each exercise was performed for 5 repetitions, for 2 sets for first 3 weeks and then progressed to 10 repetitions, 2 sets for next 3 weeks. After these exercises were done, the subjects were made to do cool down exercises for 5 minutes. The intervention protocol was as follows.

\section{INTERVENTION}

WEEK 0-3

\begin{tabular}{|}
\begin{tabular}{|c|c|c|c|}
\hline Sr.no & Exercises & Repetitions & Sets \\
\hline $\mathbf{1}$ & Sit to stand & 5 & 2 \\
\hline $\mathbf{2}$ & Calf raises & 5 & 2 \\
\hline $\mathbf{3}$ & Knee -ups & 5 & 2 \\
\hline $\mathbf{4}$ & Partial crunches & 5 & 2 \\
\hline $\mathbf{5}$ & Alternating superman & 5 & 2 \\
\hline Sr.no & Exercises & Repetitions & Sets \\
\hline $\mathbf{1}$ & Sit to stand & 10 & 2 \\
\hline $\mathbf{2}$ & Calf raises & 10 & 2 \\
\hline $\mathbf{3}$ & Knee -ups & 10 & 2 \\
\hline $\mathbf{4}$ & Partial crunches & 10 & 2 \\
\hline $\mathbf{5}$ & Alternating superman & 10 & 2 \\
\hline
\end{tabular}
\end{tabular}

\section{RESULT}

Demographic Data: Table 1 and figures 1a and $1 \mathrm{~b}$ reflect that the mean age of subjects in the present study was $71.22 \pm 4.438$. Out of 60 subjects who were included in our study, $75 \%$ were females whereas $25 \%$ were males.

Bergs balance scale: Table 2 and figure 2 show statistically improvement in pre and post treatment mean values of BBS scores 47.02 and 50.47 respectively. Statistically significant improvement in pre and post treatment values of BBS scores was found with $P$ value $=<0.001$.

Performance oriented mobility assessment: improvement in pre and post treatment mean values of POMA scores 20.95 and 24.43 respectively. Statistically significant improvement in pre and post treatment values of POMA scores was found with $P$ value $=<0.001$.

Table 1 (a): Age and Gender Distribution of Subjects: $(\mathrm{N}=60)$.

\begin{tabular}{|c|c|c|c|}
\hline & Male & $\mathbf{1 5}$ & $\mathbf{2 5}$ \\
\cline { 2 - 4 } & Female & 45 & 75 \\
\hline Age (Mean \pm SD) years & \multicolumn{2}{|c|}{$71.22 \pm 4.438$} \\
\hline
\end{tabular}

Fig. 1 (a): Age Distribution of Subjects: $(N=60)$.

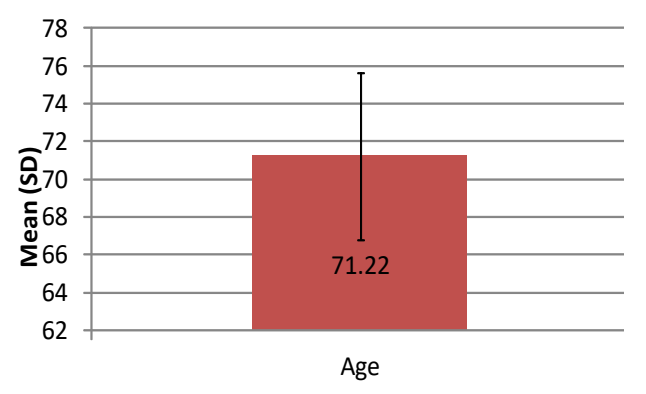

Table 2(a): Comparison of the effect of low intensity body weight training on balance using Bergs Balance Scale score in terms of $\{$ Mean (SD) $\}$ pre and post using paired $t$ test.

\begin{tabular}{|c|c|c|c|c|c|c|}
\hline & Group & $\mathrm{N}$ & Mean & $\begin{array}{c}\text { Std. } \\
\text { Deviation }\end{array}$ & t value & P value \\
\hline \multirow{2}{*}{$\begin{array}{c}\text { Bergs Balance } \\
\text { Scale }\end{array}$} & Pre & 60 & 47.02 & 1.535 & \multirow{2}{*}{12.758} & $<0.001 * *$ \\
\cline { 2 - 5 } & Post & 60 & 50.47 & 2.646 & & \multirow{2}{*}{${ }^{2}$} \\
\hline
\end{tabular}


Figure 2 (b): Comparison of the effect of low intensity body weight training on balance using Bergs Balance Scale score in terms of $\{$ Mean (SD) $\}$ pre and post using paired $t$ test.

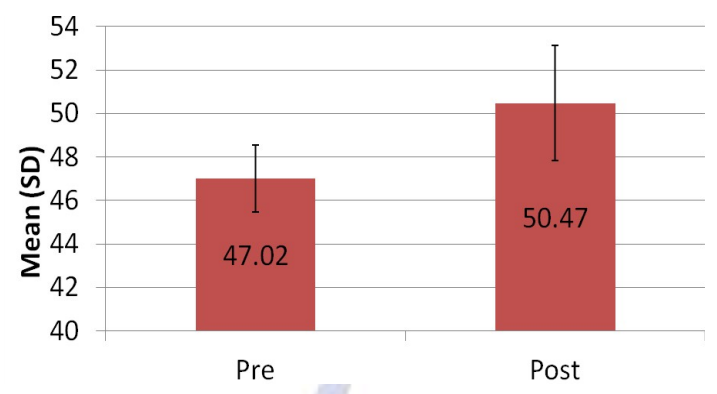

Table 3(a): Comparison of the effect of low intensity body weight training on balance using performance oriented mobility assessment(POMA) in terms of $\{$ Mean (SD) $\}$ pre and post using paired $t$ test.

\begin{tabular}{|c|c|c|c|c|c|c|}
\hline & Group & N & Mean & $\begin{array}{c}\text { Std. } \\
\text { Deviation }\end{array}$ & t value & P value \\
\hline Performance Oriented & Pre & 60 & 20.95 & 1.76 & \multirow{2}{*}{17.212} & $<0.001 * *$ \\
\cline { 2 - 6 } Mobility Assessment & Post & 6 & 24.43 & 1.845 & & \\
\hline
\end{tabular}

Fig. 3(b): Comparison of the effect of low intensity body weight training on balance using performance oriented mobility assessment(POMA) score in terms of (Mean (SD)\} pre and post using paired t test.

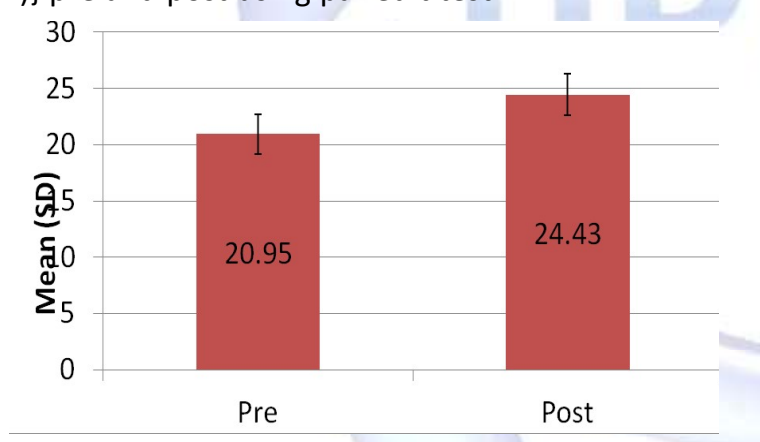

\section{DISCUSSION}

The purpose of the study was to find the effect of low intensity body weight training on balance in older adults. The study was conducted for a period of 6 weeks in which exercise sessions were given 4 times every week. Bergs balance scale and Performance Oriented mobility assessment scale were the outcome measures used.

Figure 2 demonstrates significantly improved values on Bergs Balance scale post treatment with respect to balance in older adults (pre mean 47.02 and after 6 weeks, post mean 50.47) Hence indicating a significant improvement $(p<0.05)$.

figure 3 demonstrates significantly improved values on Performance Oriented mobility assessment (POMA) post treatment with respect to balance in older adults (pre mean 20.97 and after 6weeks of training, post mean 24.43). Hence indicating a significant improvement $(p<0.05)$ in balance ability of older adults.

The primary possibility of improvement in balance in older adults could be improved muscular strength. Murat Karabulut et. al. 2010 mentioned that utilizing lower loads and lower training volumes compared to traditional resistance training resulted in similar changes in lower body skeletal muscle strength as the traditional highintensity resistance training program. It is well accepted that in training protocols, the initial rapid gain (in first 4 to 8 weeks) in the tension generating capacity of muscles is largely attributed to neural responses and neuromuscular adaptations. Hess et. al 2005 explained these adaptations such as enhanced output from supraspinal centres, altered neural drive that decreases co activation of antagonist muscles and greater activation of synergist muscles, or more effective coupling within the spinal inter neuronal pathways. Sale et. al 1998 asserts that this increased neural activation of prime movers increases motor unit synchronization and enhances activation of fast-twitch motor units, resulting in better muscle coordination and efficiency. Supporting these findings, Karabulut et. al. 2010 published an article which suggested that leg muscle strength improves with the low-load vascular restriction training and the VR-RT20 training protocol was almost as effective as the RT80 training protocol for increasing muscular strength in older men.

D. L. Sturnieks et. al 2008 published a review article highlighting the correlation between balance and strength in older adults. He concluded that the speed and organization of muscle force also contributes to balance problems in older. A reduced capacity for rapid force generation would limit the ability to respond quickly to a loss of balance and thereby increase the risk of falling. In accordance to this study Dr. R. Orr et. Al 2010 also carried out a systematic review on the association of muscle strength and muscle power to balance performance in older adults. Hence we hypothesize that increase in muscle strength could be one of the reasons that lead to improvement in balance impairment.

Another factor responsible for improvement in balance could be the improved proprioception 
According to D.LSturnicks, et. al 2008 this proprioceptive input from the lower limbs is arguably the most important contributor to maintenance of balance. The receptors responsible for detecting joint position include the Pacinian corpuscles and Ruffini end-organs found in the joint capsule and the Golgi tendon organs and muscle spindles found in the muscle. All these receptors are sensitive to changes in tension within the muscle contractions or non contractile tissues. Also, the tactile information, particularly from the soles of the feet, provides additional sensory information on force distribution during weight-bearing activities, to assist in balance control. Plantar tactile sensitivity has been shown to be reduced in older adults and correlated with measures of balance and functional test performance. Proprioception is more accurate during weight bearing due to the increase in receptor activation. Similar study conducted by Fernando Ribeiro et. al 2010 highlighted that deficits in proprioception contribute to balance disorders and the risk of falls in older people.

Limitations: Repetitive maximum (RM) was not taken into consideration before starting the intervention program. Screening for the subjects with vestibular and visual impairments was not done in our study. The history of fall was not taken into consideration.

Recommendations for further research: Future studies can correlate other parameters like cognitive function, quality of life with balance in elderly subjects. Further studies can include intervention program of low intensity weight training sessions on daily basis. Further studies can also focus on finding out long term effects of low weight training on balance.

\section{CONCLUSION}

In conclusion, the results conclude that low intensity bodyweight training protocol conducted for a period of 6 weeks has been shown to have a positive effect on the balance function in older adults.

\section{Conflicts of interest: None}

\section{REFERENCES}

[1]. Flatt T. A new definition of aging?. Frontiers in genetics. 2012 Aug 23;3:148.
[2]. Kalyani RR, Corriere M, Ferrucci L. Age-related and disease-related muscle loss: the effect of diabetes, obesity, and other diseases. The lancet Diabetes \& endocrinology. 2014 Oct 1;2(10):819-29.

[3]. Schewe CD. Marketing to our aging population: Responding to physiological changes. Journal of Consumer Marketing. 1988 Mar 1;5(3):61-73.

[4]. Boss GR, Seegmiller JE. Age-related physiological changes and their clinical significance. Western Journal of medicine. 1981 Dec;135(6):434.

[5]. Sturnieks DL, St George R, Lord SR. Balance disorders in the elderly. Neurophysiologie Clinique/Clinical Neurophysiology. 2008 Dec 1;38(6):467-78.

[6]. Jung $\mathrm{H}$, Yamasaki M. Association of lower extremity range of motion and muscle strength with physical performance of community-dwelling older women. Journal of physiological anthropology. 2016 Dec;35(1):30.

[7]. Cho KH, Bok SK, Kim YJ, Hwang SL. Effect of lower limb strength on falls and balance of the elderly. Annals of rehabilitation medicine. 2012 Jun 1;36(3):38693.

[8]. Singh F, Foster C, Tod D, McGuigan MR. Monitoring different types of resistance training using session rating of perceived exertion. International Journal of Sports Physiology and Performance. 2007 Mar;2(1):34-45

[9]. Harrison JS. Bodyweight training: A return to basics. Strength \& Conditioning Journal. $2010 \mathrm{Apr}$ 1;32(2):52-5.

[10]. Kanda K, Yoda T, Suzuki H, Okabe Y, Mori Y, Yamasaki K, Kitano H, Kanda A, Hirao T. Effects of low-intensity bodyweight training with slow movement on motor function in frail elderly patients: a prospective observational study. Environmental health and preventive medicine. 2018 Dec;23(1):4.

[11]. Mayer F, Scharhag-Rosenberger F, Carlsohn A, Cassel $M$, Müller S, Scharhag J. The intensity and effects of strength training in the elderly. Deutsches Ärzteblatt International. 2011 May;108(21):359.

[12]. Brown M, Sinacore DR, Ehsani AA, Binder EF, Holloszy JO, Kohrt WM. Low-intensity exercise as a modifier of physical frailty in older adults. Archives of physical medicine and rehabilitation. $2000 \mathrm{Jul}$ 1;81(7):960-5

[13]. Morgan RO, Virnig BA, Duque M, Abdel-Moty E, DeVito CA. Low-intensity exercise and reduction of the risk for falls among at-risk elders. The Journals of Gerontology Series A: Biological Sciences and Medical Sciences. 2004 Oct 1;59(10):M1062-7.

[14]. Watanabe Y, Tanimoto M, Oba N, Sanada K, Miyachi $M$, Ishii N. Effect of resistance training using bodyweight in the elderly: Comparison of resistance exercise movement between slow and normal speed movement. Geriatrics \& gerontology international. 2015 Dec;15(12):1270-7.

[15]. Watanabe $Y$, Madarame H, Ogasawara R, Nakazato $\mathrm{K}$, Ishii $\mathrm{N}$. Effect of very low intensity resistance training with slow movement on muscle size and strength in healthy older adults. Clinical physiology and functional imaging. $2014 \mathrm{Nov}$;34(6):463-70. 
[16]. Dhargave P, Sendhilkumar R. Prevalence of risk factors for falls among elderly people living in longterm care homes. Journal of clinical gerontology and geriatrics. 2016 Sep 1;7(3):99-103.

[17]. Binda SM, Culham EG, Brouwer B. Balance, muscle strength, and fear of falling in older adults. Experimental Aging Research. 2003 Apr 1;29(2):205-19..

[18]. Rubenstein LZ, Josephson KR, Trueblood PR, Loy S, Harker JO, Pietruszka FM, Robbins AS. Effects of a group exercise program on strength, mobility, and falls among fall-prone elderly men. The Journals of Gerontology Series A: Biological Sciences and Medical Sciences. 2000 Jun 1;55(6):M317-21.

[19]. Cardinal BJ. Assessing the physical activity readiness of inactive older adults. Adapted physical activity quarterly. 1997 Jan;14(1):65-73.

[20]. Steffen TM, Hacker TA, Mollinger L. Age-and genderrelated test performance in community-dwelling elderly people: Six-Minute Walk Test, Berg Balance Scale, Timed Up \& Go Test, and gait speeds. Physical therapy. 2002 Feb 1;82(2):128-37.

[21]. Guccione, A., Wong, R. and Avers, D. (2012). Geriatric physical therapy. St. Louis: Elsevier/Mosby.

[22]. Faber MJ, Bosscher RJ, van Wieringen PC. Clinimetric properties of the performance-oriented mobility assessment. Physical therapy. 2006 Jul 1;86(7):94454.

[23]. Kisner C, Colby L. Therapeutic exercise. 6th ed.

[24].Ehsani F, Abdollahi I, Bandpei MA, Zahiri N, Jaberzadeh $\mathrm{S}$. Motor learning and movement performance: older versus younger adults. Basic and clinical neuroscience. 2015 Oct;6(4):231.
[25]. Chiviacowsky S, Cardozo PL, Chalabaev A. Age stereotypes' effects on motor learning in older adults: The impact may not be immediate, but instead delayed. Psychology of Sport and Exercise. 2018 May 1;36:209-12.

[26]. Seidler RD. Differential effects of age on sequence learning and sensorimotor adaptation. Brain research bulletin. 2006 Oct 16;70(4-6):337-46.

[27]. Karabulut M, Abe T, Sato Y, Bemben MG. The effects of low-intensity resistance training with vascular restriction on leg muscle strength in older men. European journal of applied physiology. 2010 Jan 1;108(1):147.

[28]. Karabulut M, Abe T, Sato Y, Bemben M. Overview of neuromuscular adaptations of skeletal muscle to KAATSU Training. International Journal of KAATSU Training Research. 2007;3(1):1-9.

[29]. Sale DG. Neural adaptation to resistance training. Medicine and science in sports and exercise. 1988 Oct;20(5 Suppl):S135-45.

[30]. Hess JA, Woollacott M. Effect of high-intensity strength-training on functional measures of balance ability in balance-impaired older adults. Journal of manipulative and physiological therapeutics. 2005 Oct 1;28(8):582-90.

How to cite this article:

Sumayya Faiz Shaikh, Simran Parti, Khyati Shah, Ronika Agrawal. ROLE OF CORE STABILITY EXERCISES IN OBESE INDIVIDUALS WITH LOW BACK PAIN: A PROSPECTIVE STUDY. Int J Physiother Res 2019;7(3):3055-3060. DOI: 10.16965/ijpr.2019.116 